\title{
Models for saturation damage state and interfacial shear strengths in multilayer coatings
}

\author{
Y. Leterrier $^{\mathrm{a}, *}$, J. Waller $^{\mathrm{a}}$, J.-A.E. Månson ${ }^{\mathrm{a}}$, J.A. Nairn ${ }^{\mathrm{b}}$ \\ ${ }^{a}$ Laboratoire de Technologie des Composites et Polymères (LTC), Ecole Polytechnique Fédérale de Lausanne (EPFL), CH 1015 Lausanne, Switzerland \\ ${ }^{\mathrm{b}}$ Wood Science E Engineering, Oregon State University, Corvallis, OR 97330, USA
}

\section{A R T I C L E I N F O}

\section{Article history:}

Received 9 April 2009

Received in revised form 7 October 2009

\section{Keywords:}

Multilayer coatings

Polymer substrate

Interfacial shear strength

Shear-lag analysis

Flexible electronics

\begin{abstract}
A B S T R A C T
The present work investigates the saturation damage state of a two-layer coating on a substrate (layer 1/layer 2/substrate) under uniaxial tensile loading in order to derive expressions for the interfacial strength between layer 1 and layer 2, and between layer 2 and substrate. It is based on experimental data on specimens where layer 1 is an inorganic film, layer 2 is an organic coating and the substrate is a polymer. The analysis is relevant to the cases where layer 1 cracks first, followed by layer 2, in which cracks appear due to stress concentrations caused by the cracks in layer 1 . It considers the cases where at least one interface is completely yielded with shear stress equal to the interfacial shear stress, and where the crack density in layer 1 is equal to or higher than the crack density in layer 2. The possible situations depend on the relative shear strengths between layers 1 and 2 and between layer 2 and the substrate. The interfacial shear strength between layer 1 and layer 2, and between layer 2 and substrate are derived for elastic and yielded stress transfer cases and found to frame experimental values obtained with single-layer coatings.
\end{abstract} (c) 2009 Elsevier Ltd. All rights reserved.

\section{Introduction}

Multilayer coatings on polymer substrates are developed for an increasing number of applications ranging from optical systems to flexible electronic devices and photovoltaic modules (Chalamala et al., 2004; Crawford, 2005). A representative case of such multilayer films consists of an inorganic layer (e.g., oxide or nitride diffusion barrier) and an organic layer (e.g., acrylate-based planarization coating, so-called hard-coat, HC) deposited on a polymer substrate (Leterrier et al., 2004). Hard-coats have been developed to buffer the influence of the polymer substrates on the generation of defects during deposition of the inorganic coating, resulting in improved surface quality and increased stiffness compared to the substrate alone (Shu et al., 2007). The functional and mechanical performance of the multilayer is controlled by the cohesive prop-

\footnotetext{
* Corresponding author. Tel.: +41 21693 4848; fax: +41 216935880 .

E-mail address: yves.leterrier@epfl.ch (Y. Leterrier).
}

erties of each individual layer and the interfacial adhesion of adjacent layers.

The fragmentation test method, in which coating cracking is analyzed as a function of tensile strain has been extensively used to obtain these properties (Leterrier, 2003). The accuracy of this method is primarily related to the absence of third body interactions, such as indentercoating friction in case of scratch and indentation tests, or adherent-coating traction in case of peel and pull-out tests. The topic of multiple cracking of coatings on high elongation substrates (and matrices in composite laminates) has motivated a considerable amount of work, for instance to obtain statistical strength parameters from crack spacing distributions (Hui et al., 1999; Leterrier et al., 1997a,b; Ochiai et al., 2007) and layer toughness (Kim and Nairn, 2000; Nairn, 2000). Prior analyses of experimental data, however, are limited to single coatings (Andersons et al., 2007; Handge, 2002; Handge et al., 2000; Howells et al., 2008; Hsueh and Yanaka, 2003; Leterrier et al., 2004; Tang et al., 2001; Yanaka et al., 1999). In this case, the interfacial shear strength (IFSS, representative of 
practical adhesion) can be derived from an interfacial stress transfer analysis. Solutions for elastic stress transfer at interfaces in multiphase materials were derived using shear-lag (Cox, 1952; Mendels et al., 1999; Nairn, 1997) and variational methods (Hashin and Shtrikman, 1963; Nairn, 1992). The case of yielded interfaces was considered since in practice the interfacial shear stress may reach the yield limit (Kelly and Tyson, 1965). Models for partially yielded interfaces were also derived to account for the elasto-plastic behavior of the matrix in the case of composites (Piggott, 1980) and of the substrate in the case of multilayers (McGuigan et al., 2003). For yielded and partially yielded interfaces IFSS is proportional to the density of tensile cracks at saturation of the fragmentation process $\left(\mathrm{CD}_{\text {sat }}\right.$, i.e., when no more cracks appear as the tensile strain is increased), related to the so-called critical stress transfer length (Leterrier et al., 1997b).

For multilayer coatings the failure of adjacent layers is coupled (cracking of one of the layers influences cracking of the other layer and vice-versa), which invalidates prior stress transfer theories used to derive the IFSS. This paper considers two-layer coatings. In this case, when the first layer starts cracking, stress concentrations are induced in the second layer, thus lowering its strain to failure. Cracking of the second layer relaxes the axial stress in the first layer, which prevents further cracking of the first layer. In other words the stress partitions between the two layers, which changes the critical length to achieve failure stress in one or both layers. The problem is complicated due to the uncertainty about the stress state at the interface between the two layers.

The objective of this paper is to solve the stress transfer problem for a two-layer coating on a substrate under uniaxial tensile loading (in the order layer 1/layer 2/substrate) for specimens where layer 1 cracks first, and enable determination of the IFSS between layer 1 and layer 2, and between layer 2 and the polymer substrate. The theoretical derivation is applied to several inorganic layer/HC/polymer films representative of multilayer structures developed as substrates for flexible electronic devices.

\section{Materials and experimental methods}

\subsection{Materials}

Two main types of multilayer films were analyzed, including single-layer (layer 1/substrate) and two-layer coatings (layer 1 /layer $2 /$ substrate, which in some cases had the same layer 2 on the opposite side of the substrate). In the first type, layer 1 was a silicon nitride film (SIN) with three different thickness (300, 400 and $500 \mathrm{~nm}$ ), layer 2 was a silica-acrylate hybrid hard-coat (HC, thickness $2 \mu \mathrm{m})$ and the substrate was a $100 \mu \mathrm{m}$ thick, high temperature aromatic polyester film (ARY, Arylite, Ferrania Technologies), also coated on the opposite side with the same $2 \mu \mathrm{m}$ thick HC layer. The top HC, on the inorganic film side, is labeled here as ' $\mathrm{HC1}$ ', and the bottom $\mathrm{HC}$, located on the other side of the substrate, is labeled 'HC2'. The HC2 layer with same thickness and same thermal history as the HC1 layer will serve as a reference to quantify the influence of
SIN on HC cracking, and to obtain the IFSS between HC and the substrate. In the second type of multilayer film, layer 1 was a $209 \mathrm{~nm}$ thick oxide film (OXI), layer 2 was a HC layer with four different thicknesses (1, 2, 3, $4.5 \mu \mathrm{m})$ and the substrate was a $125 \mu \mathrm{m}$ thick polyethylene terephthalate film (PET, U34, Toray Company). HC/ PET films without the OXI layer and with the same HC thicknesses as in the two-layer coatings were also produced as references. The elastic properties of the layers relevant for the analysis developed in the following are reported in Table 1 . The Young's modulus values were obtained using nanoindentation tests of the layers deposited on glass substrates and the Poisson's ratio values were estimated. All investigated multilayer structures are listed in Table 2 .

\subsection{The fragmentation test}

In the fragmentation test, the evolution of crack patterns in the brittle coating is monitored as a function of the uniaxial tensile load applied to the substrate (Leterrier et al., 1997b). Rectangular samples with gauge length $40 \mathrm{~mm}$ and width $10 \mathrm{~mm}$ were carefully cut from the foils using a razor blade. Tests were carried out at a nominal strain rate of $4.2 \times 10^{-4} \mathrm{~s}^{-1}$ in situ in an optical microscope (Olympus BX60), using a computer-controlled tensile frame equipped with contactless video extensometry to overcome compliance effects. The coating strain at failure was measured with accuracy better than $10^{-3}$. The progressive cracking of the coating was analyzed in terms of crack density, equal to the number of tensile cracks per unit length multiplied by substrate elongation to correct, to a first approximation, for crack opening. As will be shown later, the contrast was high enough to discriminate cracks in layer 1 from cracks in layer 2. Two samples of each type of film were analyzed.

\section{Experimental results}

Fig. 1 shows the damage state in SIN and SIN/HC coatings on ARY and in OXI and OXI/HC coatings on PET at approximately $3 \%$ strain. In all cases tensile cracks perpendicular to the applied load are evident. The density of tensile cracks in the SIN and OXI layers is much higher when there is no HC layer. Cracks in the HC layer are also visible and were easily discriminated from cracks in the top layer 1 thanks to large differences in contrast, the HC cracks being much darker under reflected illumination mode. Interestingly, the HC layer systematically cracked at cracks previously formed in the SIN or OXI layers, due to stress concentrations at the tip of the crack at the SIN/HC or $\mathrm{OXI} / \mathrm{HC}$ interface. This is evident in Fig. 2, which displays

Table 1

Elastic properties of layers.

\begin{tabular}{lcl}
\hline Layer & Young's modulus [GPa] & Poisson's ratio \\
\hline SIN & 100 & 0.2 \\
OXI & 130 & 0.15 \\
HC & 6 & 0.35 \\
\hline
\end{tabular}


Table 2

$\operatorname{COS}$ and $\mathrm{CD}_{\text {sat }}$ of SIN, OXI and HC layers ('NM' for 'not measured').

\begin{tabular}{|c|c|c|c|c|c|c|c|c|}
\hline \multirow[t]{2}{*}{ Multilayer structure } & \multirow{2}{*}{$\begin{array}{l}\text { SIN/OXI layer } \\
\text { thickness [nm] }\end{array}$} & \multirow{2}{*}{$\begin{array}{l}\text { HC1/HC2 layer } \\
\text { thickness }[\mu \mathrm{m}]\end{array}$} & \multicolumn{3}{|c|}{ Crack onset strain [\%] } & \multicolumn{3}{|c|}{ Crack density at saturation $\left[\mathrm{mm}^{-1}\right]$} \\
\hline & & & Inorganic film & HC1 & $\mathrm{HC} 2$ & Inorganic film & $\mathrm{HC} 1$ & $\mathrm{HC} 2$ \\
\hline SIN/ARY & 400 & - & $1.47 \pm 0.06$ & - & - & 170 & - & - \\
\hline SIN/HC1/ARY/HC2 & 300 & 2 & $1.63 \pm 0.09$ & 2.30 & NM & 115 & 48 & NM \\
\hline SIN/HC1/ARY/HC2 & 400 & 2 & $1.36 \pm 0.13$ & 1.75 & 5.30 & 65 & 44 & 25 \\
\hline SIN/HC1/ARY/HC2 & 500 & 2 & $0.62 \pm 0.06$ & NM & NM & 70 & NM & NM \\
\hline OXI/PET & 209 & - & 0.80 & - & - & 280 & - & - \\
\hline HC1/PET & - & 1 & - & 8.7 & - & - & 62 & - \\
\hline HC1/PET & - & 2 & - & 6.6 & - & - & 54 & - \\
\hline HC1/PET & - & 3 & - & 5.8 & - & - & 37 & - \\
\hline HC1/PET & - & 4.5 & - & 4.3 & - & - & 29 & - \\
\hline $\mathrm{OXI} / \mathrm{HC} 1 / \mathrm{PET}$ & 209 & 1 & 0.72 & 2.0 & - & 150 & 121 & - \\
\hline OXI/HC1/PET & 209 & 2 & 0.73 & 1.9 & - & 120 & 110 & - \\
\hline OXI/HC1/PET & 209 & 3 & 0.80 & 1.9 & - & 95 & 70 & - \\
\hline OXI/HC1/PET & 209 & 4.5 & 0.75 & 2.6 & - & 90 & 35 & - \\
\hline
\end{tabular}
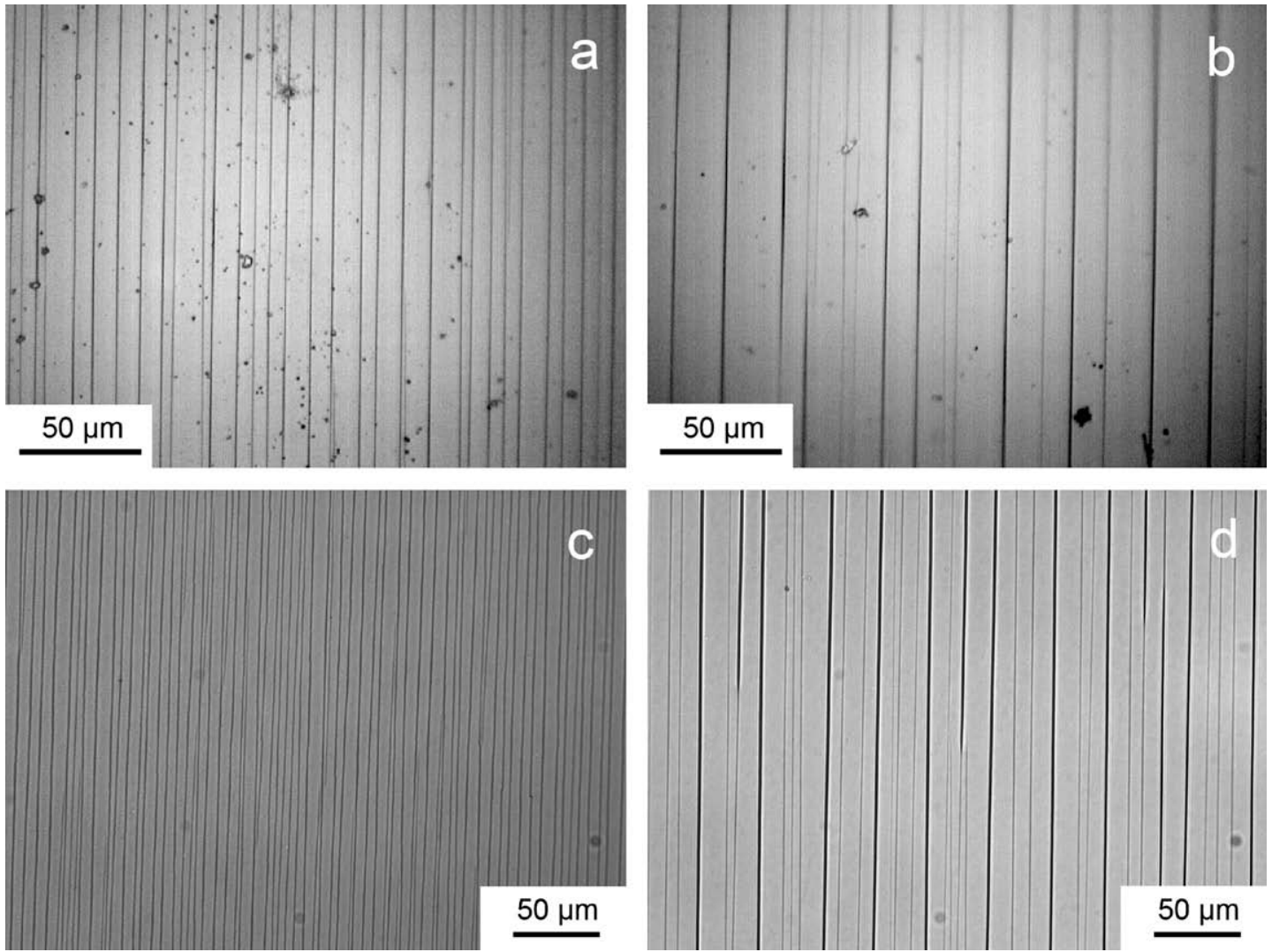

Fig. 1. Optical micrographs of tensile cracks in single-layer and two-layer coatings on ARY and PET under $3 \%$ strain; (a) 400 nm thick SIN coating on ARY; (b) $400 \mathrm{~nm}$ thick SIN coating (thin cracks) and $2 \mu \mathrm{m}$ thick HC1 layer (thicker cracks) on ARY; (c) 209 nm thick OXI coating on PET; (d) 209 nm thick OXI coating (thin cracks) and $3 \mu \mathrm{m}$ thick HC layer (thicker cracks) on PET. Several tips of HC cracks are visible, and HC cracks coincide with cracks previously formed in the SIN (b) or OXI layers (d).

a high magnification scanning electron micrograph of the cracked SIN/HC1 coating on the ARY substrate.

The fragmentation process of selected single-layer and two-layer coatings on the strained substrates is depicted in Figs. 3 and 4, in the form of crack density (CD) vs. strain data. The relevant data for the models developed in the following section are reported in Table 2. As shown in Fig. 3 the single SIN and single HC2 layers cracked at $1.5 \%$ and $5.3 \%$ strain, respectively. The SIN layer on HC1 cracked at $1.4 \%$ strain, i.e., the same as that of the single-layer within experimental scatter. In contrast, the HC1 layer with a SIN layer on top cracked at $1.8 \%$, a factor of three times lower than the single HC2 layer. As was noticed in Fig. 1, this considerable reduction in crack onset strain (COS) for the HC 


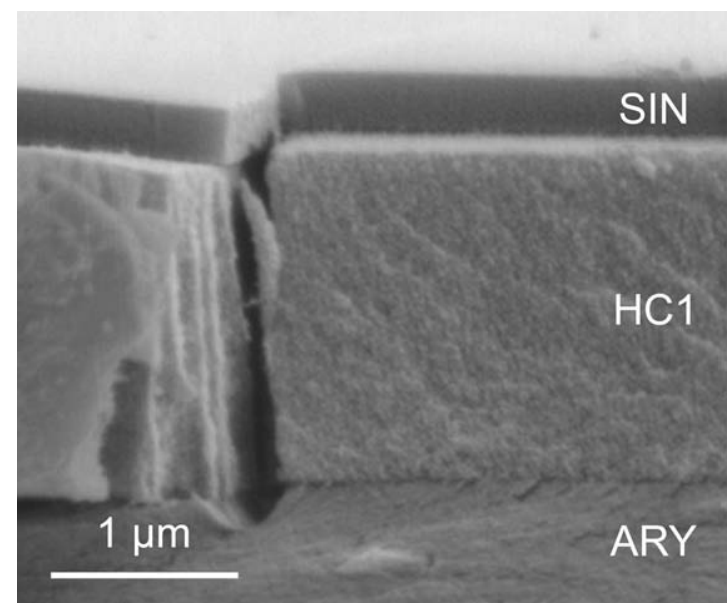

Fig. 2. Electron micrograph of a through-thickness crack in the $400 \mathrm{~nm}$ SIN/2 $\mu \mathrm{m}$ HC1 coating on ARY.

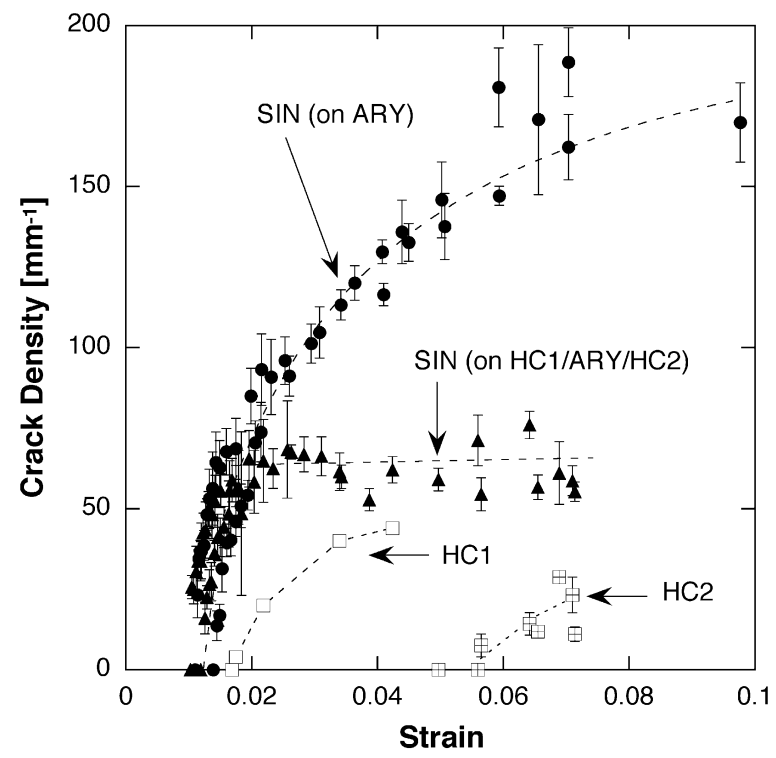

Fig. 3. Crack density vs. strain in $400 \mathrm{~nm}$ thick SIN and $2 \mu \mathrm{m}$ thick HC layers on ARY.

layer results from the presence of stress concentrations at tips of the cracks in the adjacent SIN layer. The saturation crack density of the single SIN and HC2 layers is equal to $170 \mathrm{~mm}^{-1}$ and $25 \mathrm{~mm}^{-1}$, respectively. It is evident that cracking of the SIN layer on HC1 is stopped when HC1 starts cracking, to a saturation level equal to approx. $65 \mathrm{~mm}^{-1}$. It is also evident that the saturation crack density of the HC1 layer with SIN on top is much higher $\left(44 \mathrm{~mm}^{-1}\right)$ than that of the single HC2 layer $\left(25 \mathrm{~mm}^{-1}\right)$.

The same overall behavior is observed for the OXI and HC layers in Fig. 4, and in fact for all investigated structures as reported in Table 2. The COS of the OXI layer is not markedly influenced by the presence of the HC layer. However, the COS of single $\mathrm{HC}$ layers and the $\mathrm{CD}_{\text {sat }}$ of single OXI layers are substantially reduced, and the $\mathrm{CD}_{\text {sat }}$ of the single $\mathrm{HC}$ layer is increased when these two layers are in contact.

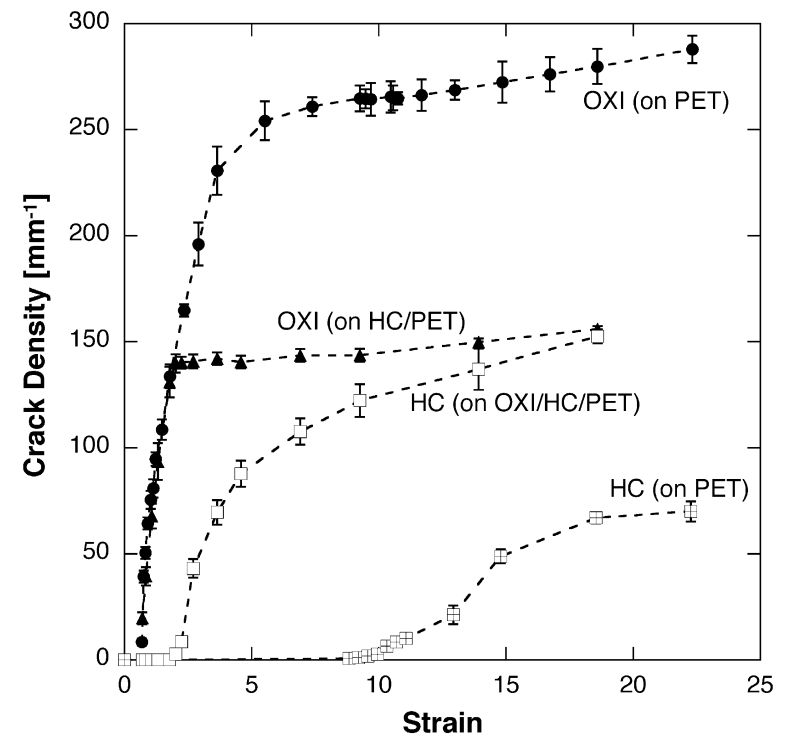

Fig. 4. Crack density vs. strain in $209 \mathrm{~nm}$ thick OXI and $3 \mu \mathrm{m}$ thick HC layers on PET.

Such considerable changes in damage behavior in the two-layer coating invalidate the classic stress transfer models used to identify the interfacial properties in single-layer coatings on substrates. A new approach that takes into account the stress transfer properties of the two interfaces in a two-layer coating on a substrate is developed as follows.

\section{Stress analysis for a substrate with two cracked coating layers}

When a single coating layer is on a substrate and the saturation damage state corresponds to shear yielding along the interface, the interfacial shear strength, $\tau_{I S S}$, is often estimated by a Kelly-Tyson (KT) analysis (Kelly and Tyson, 1965) to be

$\tau_{I S S}=\frac{2 \sigma_{u l t}^{(1)} t_{1}}{l_{c}}$

where $\sigma_{u l t}^{(1)}$ is the strength of the coating, $t_{1}$ is the thickness of the coating, and $l_{c}$ is the saturation crack spacing in the coating. Here we extend a KT approach to specimens with two coatings on a substrate. In this case, cracks in layer 1 induce stress concentrations in layer 2, thus dramatically lowering its strain to failure as was shown in Figs. 3 and 4. Cracking of layer 2 relaxes the axial stress in layer 1 in the vicinity of the crack, and the resulting exclusion zone prevents further cracking of layer 1 , hence the coupled problem. On one hand, CD in layer 1 is reduced, and in fact almost stops cracking when layer 2 cracks. On the other hand, CD in layer 2 is markedly changed compared to that without layer 1 . As a consequence Eq. (1) is not applicable in the present case with coupled fracture between the two layers.

The saturation damage state analyzed is illustrated in Fig. 5. Both the top layer (layer 1 with thickness $t_{1}$ ) and the 


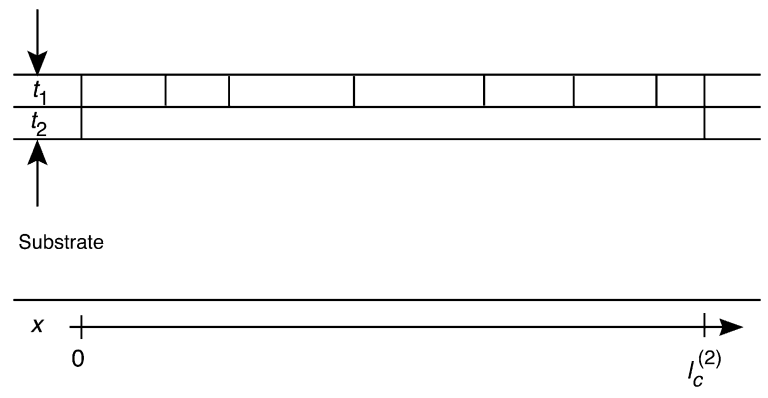

Fig. 5. Unit cell of damage for substrate with two layers. Both layer 2 and layer 1 are cracked. All cracks in layer 2 extend into layer 1 . Layer 1 may have additional cracks.

intervening layer (layer 2 with thickness $t_{2}$ ) are cracked, but layer 1 has more cracks than layer 2. Furthermore all cracks in layer 2 are assumed to continue through layer 1 resulting in a unit cell of damage as $x$ from 0 to $l_{c}^{(2)}$ where $l_{c}^{(2)}$ is the crack spacing in layer 2 . The saturation damage state in the twolayer specimen is assumed to correspond to constant shear stress between layer 2 and substrate denoted as $\tau_{2 s}$. Once the 2-S interface has yielded, additional loading no longer transmits stress into either coating and thus the damage state would no longer change.

The complication with two-layer coatings is uncertainty about the state of the 1-2 interface. The following examines two extreme possibilities, namely yielded 1-2 interface (fully yielded model) and elastic 1-2 interface (elastic-yielded model). An intermediate, partially elasticyielded model, where the 1-2 interface is partially yielded, is considered by claiming it will fall between these two limiting solutions. We consider each possibility and derive equations for $\tau_{12}$ and $\tau_{2 \mathrm{~S}}$ in terms of experimentally determined damage state parameters.

\subsection{Fully yielded model}

If the yield stress of the 1-2 interface, $\tau_{12}$, is much less than $\tau_{2 S}$, then the 1-2 interface will saturate before the $2-S$ interface and thus both interfaces will be fully yielded at constant, albeit different, shear stress. In this case, the variation in axial stress in layer $2, \sigma^{(2)}$, is found by stress equilibrium and depends on different shear stresses on either side of layer 2 (Nairn and Mendels, 2001):

$\frac{d \sigma^{(2)}}{d x}=\frac{\tau_{12}(x)-\tau_{2 S}(x)}{t_{2}}$

where the interfacial shear stresses depend on $x$ because they change sign from $-\tau$ to $+\tau$ from the left side of each fragment to the right. Layer 1 has many fragments and $\tau_{12}$ is assumed to change sign in the middle of each one. Layer 2 has a single fragment and $\tau_{2 s}$ is assumed to change sign in its middle. The resulting stress state in layer 2 is piecewise-linear as illustrated in Fig. 6 (solid line). For example, for a layer 1 fragment entirely between 0 and $l_{c}^{(2)} / 2$, Eq. (2) has constant slope of $\left(\tau_{2 s}-\tau_{12}\right) / t_{2}$ for the first half of the fragment, but because $\tau_{12}(x)$ changes sign in the middle of the fragment, the slope changes to $\left(\tau_{2 \mathrm{~S}}+\tau_{12}\right) / t_{2}$ for the second half of the fragment. A similar piecewise

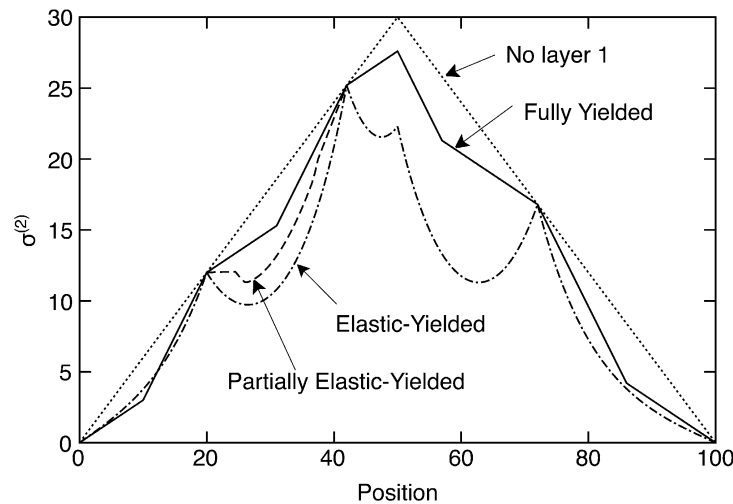

Fig. 6. Sample stress state in layer 2 for a substrate with two coating layers in the saturation damage state when the $2 \mathrm{~S}$ interface is fully yielded; the solid line is the stress state when 1-2 interface is fully yielded. The dash-dot line is when the 1-2 interface is elastic. The dashed line in one section is when the 1-2 interface is partially yielded and partially elastic. The dotted line is when layer 1 is removed.

analysis is easily derived for a fragment on either side of $l_{c}^{(2)} / 2$ or a fragment that crosses over $l_{c}^{(2)} / 2$. The dotted line is the stress state that would occur in the absence of layer 1 or if $\tau_{12}=0$. This triangular shape is the usual KT stress state with slope $\tau_{2 \mathrm{~S}} / t_{2}$ for $x<l_{c}^{(2)} / 2$ and $-\tau_{2 \mathrm{~S}} / t_{2}$ for $x>l_{c}^{(2)} / 2$.

A KT analysis finds $\tau_{\text {ISS }}$ by equating the peak stress in the coating to the strength of that layer. This approach does not work for two coatings because the peak stress in layer 2 will depend on location of the cracks in layer 1. In other words, the peak stress in layer 2 depends on more than just interfacial shear stresses. We adopted an alternative approach based on cumulative load transferred into the two layers. First, for simplicity, assume layer 1 has $n$ fragments of length $l_{c}^{(2)} / n$ (an analysis that accounts for unequal fragment lengths in layer 1 is possible, but would add unnecessary complications with little change in final results). Each fragment in layer 1 would have the triangular KT shape with peak stress equal to $\tau_{12} l_{c}^{(2)} /\left(2 t_{1}\right)$. The layer 2 stress would be given by a solid, piecewise-linear curve (see Fig. 6), but would now be symmetric about its middle due to assumption of equal fragment lengths in layer 1. By integrating the total force per unit depth carried by these stress states in the combined layers, it is easy to derive the exact relation:

$\int_{0}^{l_{c}^{(2)}}\left(t_{1} \sigma^{(1)}+t_{2} \sigma^{(2)}\right) d x=\frac{\tau_{2 S}\left(l_{c}^{(2)}\right)^{2}}{4}$

Solving for $\tau_{2 \mathrm{~S}}$ gives:

$\tau_{2 S}=\frac{4}{l_{c}^{(2)}}\left(t_{1}\left\langle\sigma^{(1)}\right\rangle+t_{2}\left\langle\sigma^{(2)}\right\rangle\right)$

where

$\left\langle\sigma^{(i)}\right\rangle=\frac{1}{l_{c}^{(2)}} \int_{0}^{l_{c}^{(2)}} \sigma^{(i)} d x$

is the average stress in a layer. Graphically, we note that $\left\langle\sigma^{(i)}\right\rangle l_{c}^{(2)}$ is the area under the layer 2 stress in Fig. 6 while 
$t_{1}\left\langle\sigma^{(1)}\right\rangle l_{c}^{(2)} / t_{2}$ is the area between the dotted triangular curve and the layer 2 stress. Next, we assumed that the saturation stress state corresponds to the state where the average stress in layer 2 reaches the same average stress it would have in the absence of layer 1 or saturation occurs when $\left\langle\sigma^{(2)}\right\rangle=\sigma_{\text {ult }}^{(2)} / 2$. For a two-layer problem, substitution into Eq. (4) gives

$\tau_{2 S}=\frac{2}{l_{c}^{(2)}}\left(t_{2} \sigma_{u l t}^{(2)}+2 t_{1}\left\langle\sigma^{(1)}\right\rangle\right)$

In the fully yielded model, the 1-2 interface is fully yielded. Thus the stress in layer 1 and the $\tau_{12}$ interfacial shear can be found by standard KT analysis and the average stress in layer 1 is given by $\left\langle\sigma^{(1)}\right\rangle=\sigma_{u l t}^{(1)} / 2$. For this model, therefore, both interfacial stresses are determined using:

$\tau_{12}=\frac{2 \sigma_{u l t}^{(1)} t_{1}}{l_{c}^{(1)}} \quad$ and

$\tau_{2 S}=\frac{2 t_{2} \sigma_{u l t}^{(2)}}{l_{c}^{(2)}}\left(1+\frac{t_{1} \sigma_{u l t}^{(1)}}{t_{2} \sigma_{u l t}^{(2)}}\right)=\frac{2 \sigma_{u l t}^{(2)} t_{2}}{l_{c}^{(2)}}+\tau_{12} \frac{l_{c}^{(1)}}{l_{c}^{(2)}}$

where $l_{c}^{(1)}$ is the crack spacing in layer 1. Eq. (7) is calculation of interfacial shear stresses based on cumulative load carried by the coatings. In the absence of layer 1 , the cumulative load approach is identical to the KT approach based on peak stress in layer 2 . In the presence of layer 1 , the cumulative load approach avoids artifacts of a peak stress analysis that cause non-physical dependence of the shear stresses on the location of the one crack in layer 1 nearest the center of layer 2 .

\subsection{Elastic-yielded model}

If $\tau_{12} \gg \tau_{2 S}$, then the 1-2 interface may be entirely elastic. In this case, the cumulative load transfer analysis still applies and thus Eq. (6) still gives $\tau_{2 \mathrm{~s}}$. The problem is thus reduced to finding $\left\langle\sigma^{(1)}\right\rangle$ by elastic stress transfer analysis between layers 1 and 2 with boundary condition of $\pm \tau_{2 \mathrm{~s}}$ applied on the surface of layer 2. This problem can be solved accurately by optimal shear-lag analysis and is a special case of the general solution derived by Nairn and Mendels (2001). For fragment $i$ in layer 1 entirely on the left of the layer 2 fragment $\left(x<l_{c}^{(2)} / 2\right)$, the general solution for the layer 1 stress is

$\sigma^{(1)}(y)=\frac{\tau_{2 S} E_{1}}{t E_{0}}\left(y+x_{i}^{0}\right)+A e^{\beta y}+B e^{-\beta y}$

where the $y$ coordinate is centered on fragment $i$ and extends from $-l_{i} / 2$ to $+l_{i} / 2, x_{i}^{0}$ is the $x$ coordinate at the center of fragment $i, t E_{0}=t_{1} E_{1}+t_{2} E_{2}, E_{1}$ and $E_{2}$ are moduli of the layers, and $\beta$ is the optimal shear-lag parameter defined by Nairn and Mendels (2001)

$\beta=\sqrt{\left(\frac{1}{t_{1} E_{1}}+\frac{1}{t_{2} E_{2}}\right) /\left(\frac{t_{1}}{3 G_{1}}+\frac{t_{2}}{3 G_{2}}\right)}$

where $G_{1}$ and $G_{2}$ are shear moduli of the layers. $A$ and $B$ are constants that are determined by setting layer 1 stress equal to zero at the cracks located at $\pm l_{i} / 2$. The result is $\sigma^{(1)}(y)=\frac{\tau_{2 S} E_{1}}{t E_{0}}\left[y+x_{i}^{0}\left(1-\frac{\cosh \beta y}{\cosh \frac{\beta l_{i}}{2}}\right)-\frac{l_{i}}{2} \frac{\sinh \beta y}{\sinh \frac{\beta l_{i}}{2}}\right]$

The interfacial shear stress can be found by differentiating $\sigma^{(1)}(y)$ (Kelly and Tyson, 1965). Our main interest is in the maximum shear stress, which occurs at the right edge of the fragment:

$\tau_{12}^{(i . \max )}=\frac{\tau_{2 S} t_{1} E_{1}}{t E_{0}}\left[-1+\beta\left(x_{i}^{0}+l_{i}\right) \tanh \frac{\beta l_{i}}{2}+\frac{\beta l_{i}}{2} \operatorname{coth} \frac{\beta l_{i}}{2}\right]$

Similar analyses can be completed for a fragment entirely on the right and for one fragment that starts on the left and ends on the right. Once the layer 1 stress is known, the stress in layer 2 can be found by force balance. An example stress state in layer 2 is shown in Fig. 6 (dashdot line). Each fragment in layer 1 has the characteristic hyperbolic shape of shear-lag solutions (Nairn and Mendels, 2001), but is skewed by the shear stress boundary condition on layer 2 .

The solution for $\tau_{2 S}$, requires integration of $\sigma^{(1)}(y)$ for each fragment and then summation over all fragments in layer 1. Integration of the single fragment in Eq. (10) gives

$\int_{0}^{l_{i}} \sigma^{(1)}(y) d y=\frac{\tau_{2 S} E_{1} x_{i}^{0} l_{i}}{t E_{0}}\left(1-\frac{2}{\beta l_{i}} \tanh \frac{\beta l_{i}}{2}\right)$

Assuming layer 1 has $n$ fragments of length $l_{c}^{(2)} / n$ and that $n$ is even leads to

$\left\langle\sigma^{(1)}\right\rangle=\frac{\tau_{2 S} E_{1} l_{c}^{(2)}}{4 t E_{0}}\left(1-\frac{2}{\beta l_{c}^{(1)}} \tanh \frac{\beta l_{c}^{(1)}}{2}\right)$

Relaxing the assumption of even and periodic fragments in layer 1 complicates the analysis, but does not significantly change the final result. Substitution into Eq. (6) gives:

$\tau_{2 S}=\frac{2 \sigma_{u l t}^{(2)} t_{2}}{l_{c}^{(2)}}\left(\frac{t_{1} E_{1}+t_{2} E_{2}}{t_{2} E_{2}+t_{1} E_{1} \frac{2}{\beta l_{c}^{(1)}} \tanh \frac{\beta l_{c}^{(1)}}{2}}\right)$

Because the 1-2 interface has not failed, this case cannot determine $\tau_{12}$. If the interface is purely elastic, however, it implies that $\tau_{12}$ is greater than the maximum shear stress seen on any fragment end. By Eq. (9), the maximum shear stress occurs near $x=l_{c}^{(2)} / 2$ implying that

$\tau_{12} \geqslant \frac{\tau_{2 S} t_{1} E_{1}}{t E_{0}}\left[-1+\frac{\beta l_{c}^{(2)}}{2} \tanh \frac{\beta l_{c}^{(1)}}{2}+\frac{\beta l_{c}^{(1)}}{2} \operatorname{coth} \frac{\beta l_{c}^{(1)}}{2}\right]$

Finally, the actual damage state might correspond to the 12 interface being partially yielded. In this case a region near the ends of the fragments would show linear variation in stress and the region in the center would show the hyperbolic shape. Such stress states can be modeled and a schematic view of the solution is shown in the second layer 1 fragment in Fig. 6 as a dashed line. Since the key term needed to find $\tau_{2 \mathrm{~S}}$ is the area between the layer 2 curve and the dotted triangular curve, the sample stress state suggests that $\tau_{2 s}$ for the partially elastic model should fall between the results for the fully yielded and the elastic-yielded model. We thus claim that $\tau_{2 \mathrm{~S}}$ is between the 
results in Eqs. (7) and (14) and that $\tau_{12}$ is greater than the minimum of the two values in Eqs. (7) and (15). Because it is not certain how much of the 1-2 interface has yielded, the experiments do not provide an upper bound to $\tau_{12}$.

\subsection{Comparison with experimental interfacial shear strength data}

The shear strengths of the two interfaces ( $\tau_{12}$ and $\tau_{2 \mathrm{~S}}$ ) in all investigated multilayers, and for the fully yielded and elastic-yielded models are shown in Figs. 7 and 8. The results for $\tau_{12}$ are compared with the IFSS value derived using standard KT analysis for the single SIN and single OXI layers on the polymer substrate (i.e., without any HC layer), $\tau_{1 s}$. This value is considered to be representative of $\tau_{12}$ since similar interfacial interactions between the vapor-formed inorganic films and the organic HC and polymer substrates are expected. The values of $\tau_{1 \mathrm{~s}}$ are close to $100 \mathrm{MPa}$, which is indeed comparable to values reported for similar inorganic coatings (Andersons et al., 2007; Howells et al., 2008; McGuigan et al., 2003; Yanaka et al., 1999). The fully yielded and elastic-yielded $\tau_{12}$ values differ by an order of magnitude, with $\tau_{1 \mathrm{~s}}$ being indeed higher than the lowest, fully yielded value. As indicated above the present approach does not provide an upper bound to $\tau_{12}$ since it is not known if that interface has reached saturation once the 2-S interface has stopped transferring additional stress.

The results for $\tau_{2 \mathrm{~S}}$ are compared to the benchmark values obtained with single HC layers, and to the KT approximation obtained using Eq. (1) on the CD of the HC layer

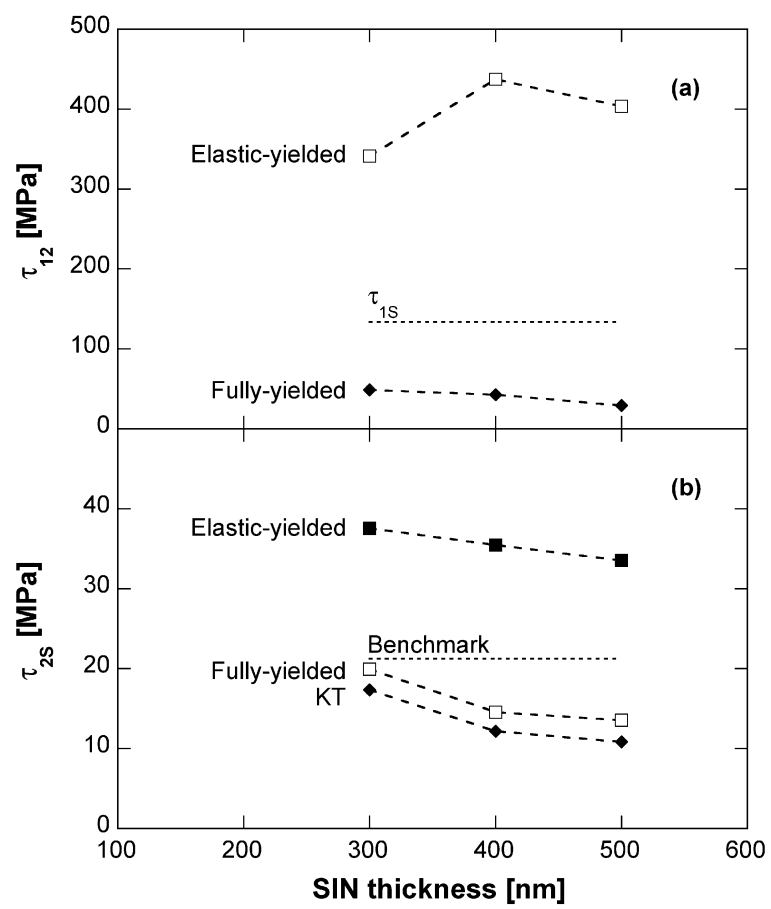

Fig. 7. IFSS between SIN and HC (a) and between HC and ARY (b), for 3 different SIN layer thickness, and for the fully yielded and elastic-yielded models. The values of $\tau_{1 \mathrm{~S}}$, KT approximation for $\tau_{2 \mathrm{~S}}$ and benchmark $\tau_{2 \mathrm{~S}}$ are also shown (see text for details).

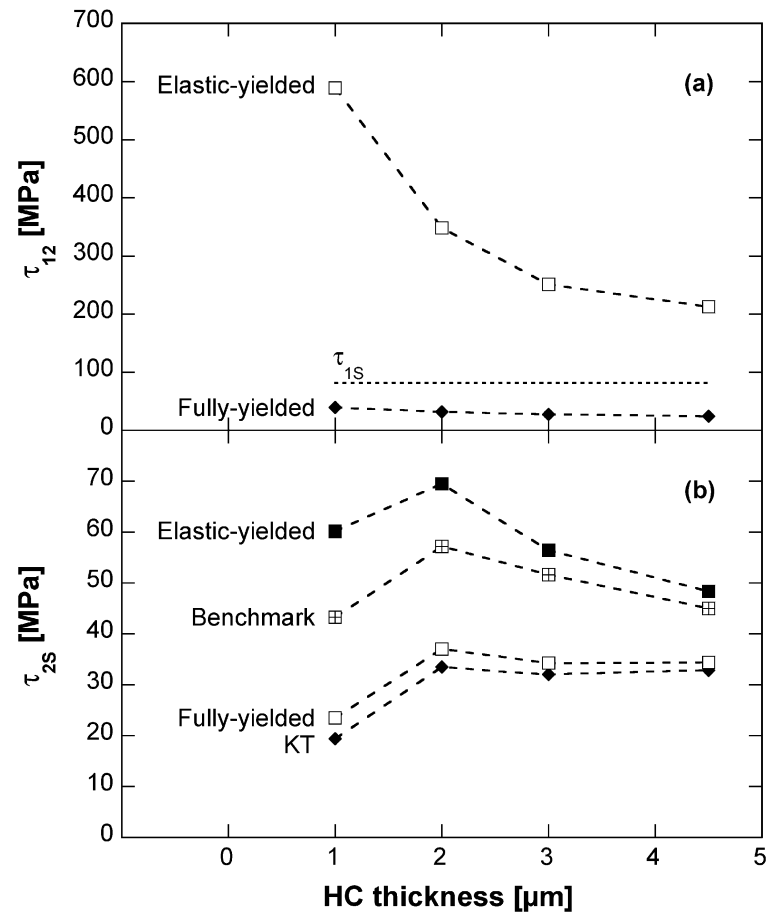

Fig. 8. IFSS between OXI and HC (a) and between HC and PET (b), for 4 different HC layer thickness, and for the fully yielded and elastic-yielded models. The values of $\tau_{1 \mathrm{~s}}$, KT approximation for $\tau_{2 \mathrm{~S}}$ and benchmark $\tau_{2 \mathrm{~s}}$ are also shown (see text for details).

in the two-layer specimens. In the case of the SIN/HC/ ARY the benchmark $\tau_{2 \mathrm{~S}}$ is close to $20 \mathrm{MPa}$, which is lower than the $\tau_{12}$ values (there is only one benchmark $\tau_{2 \mathrm{~s}}$ value since it is the SIN, which was the variable with different thicknesses, and only one HC was used). The situation differs for the OXI/HC/PET case with a benchmark $\tau_{2 \mathrm{~S}}$ comparable to $\tau_{12}$ (there are four different benchmark $\tau_{2 \mathrm{~s}}$ values since four different HC thicknesses were tested). Delamination is therefore expected to occur at sufficiently high strain at the HC/ARY interface, but not at the other interfaces. This is confirmed in Fig. 9, where extensive buckling is observed only in the case of the HC/ARY interface. It is moreover clear that the KT approximation underestimates the benchmark value by a factor of approximately 2 , which is aggravated with increasing SIN layer thickness. This confirms that the KT Eq. (1) is quite inaccurate in the case of two-layer coatings with cracks in both layers. It is also evident that the fully yielded and elastic-yielded models frame the benchmark values, and that the average of these two bounds provide a reasonable estimate of the strength of the HC/polymer interface.

\section{Conclusions}

Models to derive the two IFSS values from the tensile damage state in a two-layer coating on a polymer substrate (layer 1/layer 2/substrate) were developed assuming that the layer 2/substrate interface was yielded. The analysis considered either yielded or elastic stress transfer between 

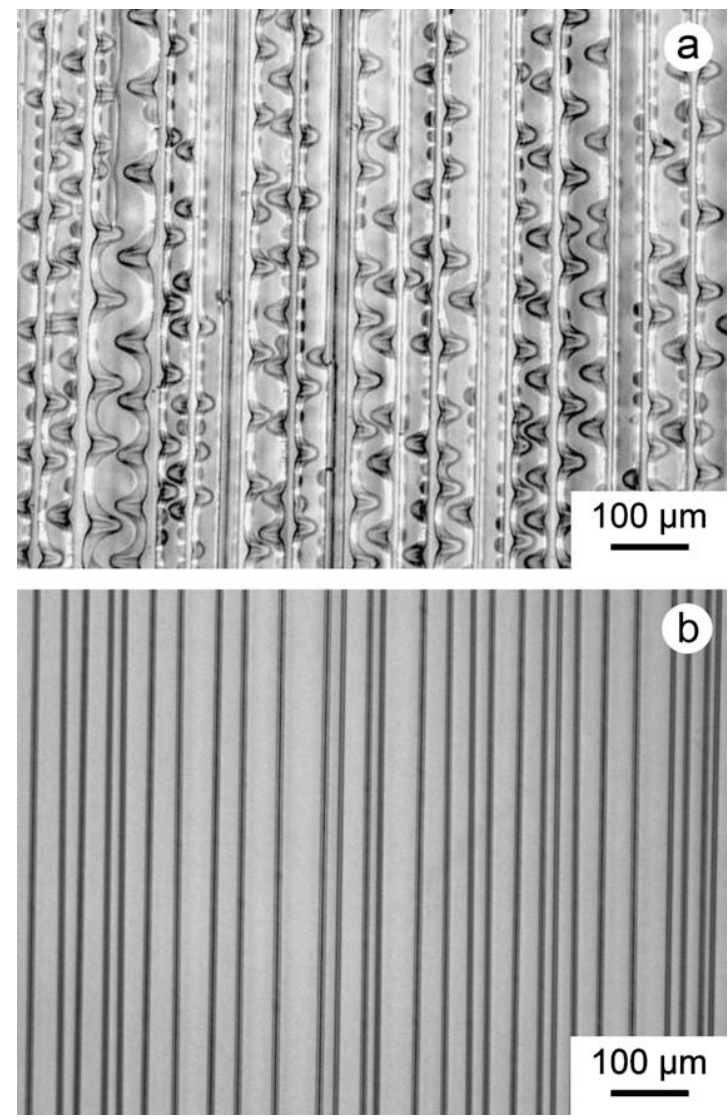

Fig. 9. Saturation damage state in $2 \mu \mathrm{m}$ thick HC on ARY (a) and $3 \mu \mathrm{m}$ thick HC on PET (b).

layer 1 and layer 2 and was applied to several nitride and oxide films on organic hard-coats. The IFSS values derived for the two interfaces from the analysis of fragmentation data were compared to the classic Kelly-Tyson IFSS and to benchmark IFSS values obtained using single-layer coatings.

In the case of the layer 2/substrate interface the fully yielded and elastic-yielded models provided lower and upper bounds to the IFSS, respectively. The KT IFSS was also found to underestimate the benchmark values by a factor of approximately 2 . The theoretical results were validated with the benchmark IFSS data and with the comparison of delamination behavior between the different hardcoats. A further model, where the 1-2 interface was partially yielded was proposed, leading to an IFSS claimed to fall between the result of the fully yielded and elasticyielded models.

In the case of the layer 1/layer 2 interface the minimum of these two models provided a lower bound to the IFSS, but no upper bound could be established due to the uncertainty about the state of that interface.

\section{Acknowledgements}

The authors acknowledge the companies Ferrania Imaging Technologies, Unaxis Balzers Ltd. (Displays Division) and Essilor International for the supply of the films samples and for financial support. They also thank Léonard Médico and the EPFL center for electron microscopy for experimental support.

\section{References}

Andersons, J., Leterrier, Y., Tornare, G., Dumont, P., Månson, J.-A.E., 2007. Evaluation of interfacial stress transfer efficiency by coating fragmentation test. Mech. Mater. 39, 834-844.

Chalamala, B.R., Gnade, B.E., Fruehauf, N., Jang, J. (Eds.), 2004. In: Flexible Electronics 2004-Materials and Device Technology. MRS Symposium Proceedings, vol. 814, Materials Research Society, Warrendale, PA.

Cox, H.L., 1952. The elasticity and strength of paper and other fibrous materials. Br. J. Appl. Phys. 3, 72-79.

Crawford, G.P. (Ed.), 2005. Flexible Flat Panel Displays. John Wiley \& Sons, Chichester, England.

Handge, U.A., 2002. Analysis of a shear-lag model with nonlinear elastic stress transfer for sequential cracking of polymer coatings. J. Mater. Sci. 37, 4803-4810.

Handge, U.A., Leterrier, Y., Rochat, G., Sokolov, I.M., Blumen, A., 2000. Two scaling domains in multiple cracking phenomena. Phys. Rev. E 62, 7807-7810.

Hashin, Z., Shtrikman, S., 1963. A variational approach to the theory of the elastic behaviour of multiphase materials. J. Mech. Phys. Sol. 11, 127140 .

Howells, D.G., Henry, B.M., Leterrier, Y., Månson, J.-A.E., Madocks, J., Assender, H.E., 2008. Mechanical properties of $\mathrm{SiO}_{x}$ gas barrier coatings on polyester films. Surf. Coat. Technol. 202, 3529-3537.

Hsueh, C.H., Yanaka, M., 2003. Multiple film cracking in film/substrate systems with residual stresses and unidirectional loading. J. Mater. Sci. 38, 1809-1817.

Hui, C.Y., Shia, D., Berglund, L.A., 1999. Estimation of interfacial shear strength: an application of a new statistical theory for single fiber composite test. Compos. Sci. Technol. 59, 2037-2046.

Kelly, A., Tyson, W.R., 1965. Tensile properties of fibre-reinforced metals: copper/tungsten and copper/molybdenum. J. Mech. Phys. Sol. 13, 329-350.

Kim, S.R., Nairn, J.A., 2000. Fracture mechanics analysis of coating/ substrate systems Part I: Analysis of tensile and bending experiments. Eng. Fract. Mech. 65, 573-593.

Leterrier, Y., 2003. Durability of nanosized gas barrier coatings on polymers. Prog. Mater. Sci. 48, 1-55.

Leterrier, Y., Andersons, J., Pitton, Y., Månson, J.-A.E., 1997a. Adhesion of silicon oxide layers on poly(ethylene terephthalate). II: Effect of coating thickness on adhesive and cohesive strengths. J. Polym. Sci. B: Polym. Phys. 35, 1463-1472.

Leterrier, Y., Boogh, L., Andersons, J., Månson, J.-A.E., 1997b. Adhesion of silicon oxide layers on poly(ethylene terephthalate). I: Effect of substrate properties on coating's fragmentation kinetics. J. Polym. Sci. B: Polym. Phys. 35, 1449-1461.

Leterrier, Y., Médico, L., Demarco, F., Månson, J.-A.E., Escola-Figuera, M., Kharrazi-Olsson, M., Betz, U., Atamny, F., 2004. Mechanical integrity of transparent conductive oxide films for flexible polymer-based displays. Thin Solid Films 460, 156-166.

McGuigan, A.P., Briggs, G.A.D., Burlakov, V., Yanaka, M., Tsukahara, Y., 2003. An elastic-plastic shear lag model for fracture of layered coatings. Thin Solid Films 424, 219-223.

Mendels, D.-A., Leterrier, Y., Månson, J.-A.E., 1999. Stress transfer model for single fibre and platelet composites. J. Compos. Mater. 33, 15251543.

Nairn, J.A., 1992. A variational mechanics analysis of the stresses around breaks in embedded fibers. Mech. Mater. 12, 131-154.

Nairn, J.A., 1997. On the use of shear-lag methods for analysis of stress transfer unidirectional composites. Mech. Mater. 26, 63-80.

Nairn, J.A., 2000. Matrix microcracking in composites. In: Kelly, A., Zweben, C. (Eds.), Comprehensive Composite Materials, PolymerMatrix Composites, vol. 2. Elsevier, Oxford, pp. 403-432 (Chapater 12).

Nairn, J.A., Mendels, D.A., 2001. On the use of planar shear-lag methods for stress transfer analysis of multilayered composites. Mech. Mater. 33, 335-362.

Ochiai, S., Iwamoto, S., Nakamura, T., Okuda, H., 2007. Crack spacing distribution in coating layer of galvannealed steel under applied tensile strain. ISIJ Int. 47, 458-465.

Piggott, M.R., 1980. Load Bearing Fiber Composites - Reinforcement Processes. Pergamon, Elmsford, NY. 
Shu, C.H., Chiang, H.C., Tsiang, R.C.C., Liu, T.J., Wu, J.J., 2007. Synthesis of organic-inorganic hybrid polymeric nanocomposites for the hard coat application. J. Appl. Polym. Sci. 103, 3985-3993.

Tang, H.X., Foran, B., Martin, D.C., 2001. Quantitative measurement of adhesion between polypropylene blends and paints by tensile mechanical testing. Polym. Eng. Sci. 41, 440-448.
Yanaka, M., Miyamoto, T., Tsukahara, Y., Takeda, N., 1999. In-situ observation and analysis of multiple cracking phenomena in thin glass layers deposited on polymer-films. Compos. Interf. 6, 409-424. 